\title{
Arbor
}

\section{Incentivos económicos de la natalidad}

Margarita Delgado

Arbor CLXXVI, 694 (Octubre 2003), 269-282 pp.

\section{Introducción}

La natalidad es una de las variables que determinan el crecimiento de una población. Las otras son la mortalidad y el saldo migratorio, el cual se obtiene por diferencia entre emigrantes e inmigrantes. La evolución de todas ellas no sólo determina el volumen sino también la estructura de esa población, lo que puede constituir un problema cuando se produce un desequilibrio.

Recientemente, los poderes públicos, los medios de comunicación, así como otras instancias -entre ellas Naciones Unidas y los institutos de investigación que elaboran proyecciones- vienen llamando la atención acerca de la evolución de dos de estas variables: la natalidad o, más propiamente, la fecundidad, y las corrientes migratorias que, desde los países menos desarrollados, se producen hacia los más desarrollados. $\mathrm{Y}$ todo ello con preocupaciones bien diferentes según se trate de un grupo u otro de países: en el caso de los menos desarrollados, con la preocupación por la alta tasa de fecundidad, — si bien no olvidando los avances producidos en las últimas décadas respecto a la reducción de la misma- y, asimismo, constatando los escasos progresos de desarrollo real que obligan a sus ciudadanos a buscar empleo en otros lugares. En el caso de los países desarrollados, las preocupaciones son las contrarias: la baja fecundidad pone en peligro la reposición de las generaciones y rompe el equilibrio anterior respecto a la estructura por edades de su pirámide poblacional, que más que pirámide parece un cono. Por lo que hace a las migraciones, el problema que se presenta 
es la capacidad de su mercado de trabajo y de la organización social en general para absorber todos los contingentes que llaman a su puerta.

Pero este trabajo tiene como objetivo centrarse en la natalidad, aunque el análisis tomará como variable la fecundidad, concepto que es un poco diferente, pero que permite medir más ajustadamente la evolución del fenómeno ${ }^{1}$. Se centrará en las características de la fecundidad en los países desarrollados, y cuando haya que recurrir a las cifras concretas se tomará como referencia el caso de España, por ser en la actualidad uno de los países con la tasa más baja del mundo desarrollado.

\section{Algunas teorías sobre la fecundidad}

Entre las diversas teorías acerca de la evolución de la fecundidad, destaca la teoría de la transición demográfica, así como algunas teorías provenientes del campo de la Economía, pero también de la Sociología y la Psicología, entre otras disciplinas. En todas ellas se trata de explicar el porqué del comportamiento reproductivo aludiendo a cuestiones económicas, sociales, psicológicas o de otra índole. Se intentan dar explicaciones plausibles del comportamiento humano en esta materia y, de hecho, uno de los enfoques más influyentes de los formulados en la segunda mitad del siglo XX hace alusión al enfoque económico del comportamiento humano (Becker, 1980).

Desde la Escuela de Chicago, a la que pertenece Becker, otros autores ponen su acento en que el hecho de tener un hijo forma parte de un proceso de toma de decisiones (Leibenstein, 1981). Desde hace algunas décadas - aproximadamente desde los años sesenta, ya que la evolución difiere entre unos y otros países- ese proceso se ha vuelto más complejo debido a varios factores, pero dos son determinantes: por un lado, el perfeccionamiento de los métodos anticonceptivos y su extensión y, por otro, la mayor tasa de actividad de las mujeres.

Las mejoras experimentadas por la tecnología de la anticoncepción hacen posible una planificación tanto del número de hijos que se quiere tener como de su espaciamiento. Esto representa una extraordinaria novedad respecto a épocas precedentes y pone al servicio de las motivaciones un instrumento de gran precisión para planificar la reproducción. Esta planificación ha devenido un notable envejecimiento del calendario de la maternidad. Un trabajo de Bosveld (1996) pone de relieve cómo los bajos índices de fecundidad alcanzados en Europa en los años ochenta y noventa, pese a las divergencias entre países respecto 
a los patrones de comportamiento, tienen que ver con el retraso de la maternidad y la infecundidad voluntaria, que, más tarde, puede transformarse en involuntaria si el retraso se prolonga.

La mayor tasa de actividad de las mujeres hace conveniente un menor número de hijos, a efectos de combinar vida familiar y laboral. Además, el coste de oportunidad que representan los hijos se ha incrementado notablemente con la participación femenina en el mercado de trabajo, por lo que muchas veces entran en contradicción ambas apetencias: tener hijos $-\mathrm{o}$ al menos un cierto número de ellos- y tener un trabajo extradoméstico. Lo de tener un trabajo extradoméstico parece una decisión bastante incontestable conforme se suceden las generaciones, pues no en vano las nuevas cohortes de mujeres han accedido a la educación en igual medida que sus congéneres masculinos $\mathrm{y}$ aspiran a formar parte de la fuerza de trabajo en similares condiciones. Además, las mujeres cada vez son más conscientes de que la autonomía personal pasa por la independencia económica. Por tanto, hay procesos que ya se admiten como irreversibles y que nadie pone en cuestión, cual es la incorporación de las mujeres al mundo del trabajo.

Tener hijos influencia y modifica nuestras vidas. Esto afecta a cuestiones económicas pero también a estilos de vida. La atención que requiere un niño a lo largo de su proceso de crecimiento exige una gran cantidad de tiempo y esfuerzo por parte de sus padres y, muy especialmente, por parte de su madre. Todavía determinados aspectos de la crianza no son compartidos de forma equitativa entre los dos miembros de la pareja y la constatación de esta circunstancia es otro elemento que las mujeres tienen en cuenta en el proceso de toma de decisiones respecto a ser madres o no.

Existen multitud de factores a considerar cuando se abordan los determinantes de la fecundidad, si bien predominan los de carácter económico, principalmente aquellos relacionados con la actividad laboral de los hipotéticos progenitores y, más específicamente, de las mujeres. Gustaffson (2001) reflexiona y teoriza sobre la importancia del retraso de la maternidad y la infecundidad voluntaria en Europa de un lado, y los condicionantes que impone la actividad laboral por otro, en términos de carrera profesional de las mujeres y rentabilidad de las inversiones en capital humano. Se trata de la ya vieja dicotomía abordada anteriormente por otros autores respecto al coste de oportunidad que representan los hijos, creciente en relación directa al nivel educativo. Pone de manifiesto cómo el retraso de la maternidad está determinado por la consecución de una carrera profesional y cómo ese retraso tiene varias consecuencias: demográficas, ya que el retraso del calendario 


\section{Margarita Delgado}

de la fecundidad acaba afectando a la intensidad de la misma - como ya indicara Bosveld-, y económicas, por cuanto la fecundidad tardía suele tener costes médicos más elevados para la madre y para el hijo. Habría que señalar también que, a largo plazo, los costes económicos de una relación desfavorable en determinados períodos de tiempo entre trabajadores activos y perceptores de subsidios es una de las principales consecuencias que enfatizan los análisis económicos del descenso de la fecundidad.

Desde el comienzo de la transición de la fecundidad en Europa, la percepción social ha ido cambiando como consecuencia de cambios que se habían ido produciendo en el sistema de valores, partiendo desde la enorme importancia en el valor del niño, como señala Ariès (1981), hasta una progresiva instalación del beneplácito social respecto a la infecundidad voluntaria o el retraso de la maternidad en años recientes. Eran tiempos en que si bien las tasas de fecundidad descendieron en toda Europa, no se pensaba que tal comportamiento sería tan persistente y se llegaría a los niveles que hemos alcanzado. Hoy, cuando las voces de alerta demográfica han sonado, es posible que la presión social cambie de signo y se acentúe sobre las parejas que no tienen hijos. Pero ¿hasta qué punto? He ahí la clave del problema. Parece obvio que no cabe pensar en un retorno a las cifras del pasado, de tres, cuatro o más hijos, anterior al trabajo fuera del hogar de la mujer, pero el nudo gordiano no está tanto en aceptar una reducción del número de hijos como situar el umbral en un punto en el que ese reducido número no sea incompatible con un crecimiento de la población - aunque sea a bajas tasas- o, por lo menos, con un cierto equilibrio en la estructura por edades.

La división sexual de las tareas, en el pasado era funcional para la sociedad: el hombre procuraba el sustento en el ámbito extradoméstico, mientras que la mujer se encargaba del cuidado de la familia, sin olvidar que también colaboraba en la producción de bienes en las economías agrícolas. Con la industrialización y, sobre todo, con la extensión de la educación, esos equilibrios se quebraron. Caldwell (1981) resume muy bien los cambios que hicieron que la alta fecundidad empezase a percibirse como una desventaja y de ahí, la imparable puesta en marcha de su reducción.

\section{La realidad de las cifras}

A efectos de proporcionar una idea de la magnitud de los cambios, en la tabla 1 se puede apreciar la evolución de algunos indicadores 
en España, que sirven de referencia para ilustrar lo sucedido con la fecundidad en Europa, de manera general. Bien es verdad que la cronología difiere y que algunas variaciones han sido más rápidas y de mayor calado en nuestro país, pero ello no desvirtúa el signo de la tendencia general.

Tabla 1

Tasas de Fecundidad (tanto por uno) por rango de nacimiento. España, 1975-99

\begin{tabular}{|c|c|c|c|c|c|c|}
\hline AÑOS & RANGO 1 & RANGO 2 & RANGO 3 & RANGO 4 & RANGO 5+ & $\begin{array}{r}\text { TOTAL } \\
\text { (I.S.F.) } \\
\end{array}$ \\
\hline 1975 & 1,008 & 0,837 & 0,481 & 0,228 & 0,226 & 2,780 \\
\hline 1980 & 0,897 & 0,693 & 0,343 & 0,148 & 0,129 & 2,210 \\
\hline 1985 & 0,725 & 0,529 & 0,227 & 0,089 & 0,067 & 1,637 \\
\hline 1990 & 0,672 & 0,480 & 0,151 & 0,046 & 0,028 & 1,377 \\
\hline 1995 & 0,600 & 0,436 & 0,110 & 0,026 & 0,015 & 1,187 \\
\hline 1999 & 0,625 & 0,442 & 0,102 & 0,021 & 0,011 & 1,203 \\
\hline $\begin{array}{l}\text { VARIACIÓN } \\
\text { 1975-99 (\%) }\end{array}$ & $-38,00$ & $-47,19$ & $-78,79$ & $-90,79$ & $-95,13$ & $-56,73$ \\
\hline
\end{tabular}

Fuente: I.N.E. y elaboración propia.

Entre 1975 y 1999 el promedio de hijos por mujer pasó de 2,78 a 1,2 , en una tendencia prácticamente descendente durante todo el período, salvo las oscilaciones de la segunda parte de los noventa que finalizan en un levísimo repunte (de 1,19 a 1,20). Si se observa la tasa de cada uno de los órdenes, se aprecia que en dicho período los hijos de rango superior a tres - cuartos, quintos y más- casi han desaparecido, pues la reducción supera el $90 \%$. Incluso la tasa de orden tres ha descendido cerca de un $80 \%$, mientras que las de uno y dos lo han hecho un $38 \%$ y un $47 \%$ respectivamente. Lo que ponen de manifiesto estos datos es que el descenso de la fecundidad es generalizado cualquiera que sea el rango que se considere, pero si se miran las cifras con más atención se puede apreciar que el leve repunte en el promedio de hijos por mujer que se produjo después de 1995 se debe exclusivamente a las tasas de orden uno y dos, que aumentan 
respectivamente $4,16 \%$ y $1,38 \%$. Es decir, que los datos evidencian que la mayor parte del reciente incremento es atribuible a mujeres que se han decidido a ser madres por primera vez, lo que puede interpretarse como una asunción de la maternidad pospuesta, ya que se produce entre las mujeres que superan los treinta años.

Entre 1975 y 1999 la aportación de los nacimientos de primer rango al total de los nacimientos pasó de $36,25 \%$ a $52,01 \%$, mientras que los de rango cuatro y superiores pasaron de $16,35 \%$ a $2,74 \%$ (tabla 2). En la medida en que unos rangos reducen su aportación, obviamente, otros la incrementan, pero es de destacar que más de la mitad de los nacidos que se produjeron en 1999 eran primeros hijos y algo más de un tercio, segundos. Esto lleva a que solamente uno de cada diez nacidos era de rango superior a dos para las mujeres que fueron madres en ese año.

Tabla 2

Aportación (\%) de los diferentes rangos al índice sintético de fecundidad España, 1975-99

\begin{tabular}{lrrrrrr} 
AÑOS & RANGO 1 & RANGO 2 & RANGO 3 & RANGO 4 & RANGO 5 & TOTAL \\
\hline $\mathbf{1 9 7 5}$ & 36,25 & 30,10 & 17,31 & 8,21 & 8,14 & $\mathbf{1 0 0}$ \\
$\mathbf{1 9 8 0}$ & 40,57 & 31,35 & 15,53 & 6,70 & 5,84 & $\mathbf{1 0 0}$ \\
$\mathbf{1 9 8 5}$ & 44,30 & 32,30 & 13,89 & 5,44 & 4,07 & $\mathbf{1 0 0}$ \\
$\mathbf{1 9 9 0}$ & 48,82 & 34,89 & 10,94 & 3,34 & 2,00 & $\mathbf{1 0 0}$ \\
$\mathbf{1 9 9 5}$ & 50,55 & 36,73 & 9,26 & 2,22 & 1,23 & $\mathbf{1 0 0}$ \\
$\mathbf{1 9 9 9}$ & 52,01 & 36,78 & 8,47 & 1,79 & 0,95 & $\mathbf{1 0 0}$ \\
& & & & & & \\
VARIACIÓN & 15,76 & 6,68 & $-8,84$ & $-6,42$ & $-7,19$ & \\
$\mathbf{1 9 7 5 - 9 9}$ (puntos) & & & & & &
\end{tabular}

Fuente: I.N.E. y elaboración propia.

La edad a la primera maternidad y a la maternidad en general, salvo entre 1975 y 1980 en que se aprecia un leve rejuvenecimiento, han seguido un curso ininterrumpidamente alcista (gráfico 1). En 1975 la edad media de las madres primíparas era 25,3 años y la de las madres en general, 28,8, una diferencia de 3,5 años. En 1999, las 


\section{Incentivos económicos de la natalidad}

mujeres que fueron madres por primera vez tenían una edad media de 29 años y las madres en general, 30,7. La diferencia entre ambos indicadores se había acortado en 1,8 años, poniendo de relieve el estrechamiento producido en el tiempo dedicado a la reproducción. Otro dato relevante es que, en 1975, de las mujeres que eran madres por primera vez sólo el 11,3\% superaba los 30 años, mientras que la cifra para 1999 es del 43,74\%. Pese a algunas oscilaciones de la tasa de fecundidad - dentro de la tendencia descendente-, el retraso del calendario de la maternidad es un hecho evidente. Esto tiene repercusiones de gran calado respecto a la intensidad final alcanzada, así como a las variaciones en la estructura por edades, aún en el supuesto de una recuperación ulterior.

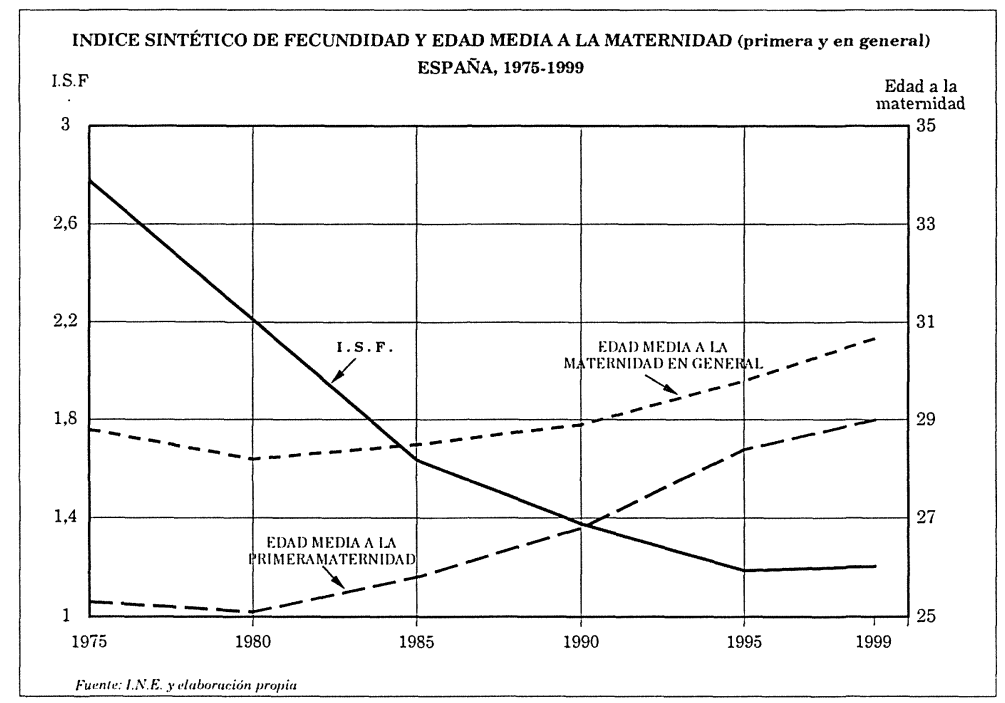

\section{Incentivos: económicos y sociales}

Cuando entre los poderes públicos y el conjunto de la ciudadanía surge la preocupación por la baja tasa de fecundidad, lo inmediato suele ser tratar de encontrar incentivos que provoquen una inversión de la tendencia en orden a atenuar los efectos negativos de la misma. En lo primero que se piensa es en los incentivos de carácter económico, algo acorde con las manifestaciones de los ciudadanos cuando en las encuestas expresan los motivos de la discrepancia entre los hijos que tienen y los que desearían tener. Pero no es un asunto sencillo ni barato, debido a que las dificultades no son coyunturales sino más bien estructurales. 


\section{Margarita Delgado}

Conviene distinguir entre las dificultades para formar una familia y las dificultades para tener hijos, porque como se apunta repetidamente (Allan, 2001), en las últimas décadas cada vez en mayor medida se ven disociados pareja y procreación. Basándose en datos sobre formación de parejas en España, se ha afirmado que el declive de la tasa de nupcialidad - que no tiene un contrapeso en las uniones consensualesincide de manera determinante sobre la tasa de fecundidad (Delgado, 2000), tanto de manera directa como indirecta. En primer lugar, si se retrasa la unión, eso mismo ya supone un retraso automático sobre la fecundidad en tanto los hijos suelen tenerse dentro de una unión conyugal. Pero, asimismo, puede dificultar la posterior recuperación debido a que el retraso afecta al número final de hijos que se tienen. Así pues, las dificultades que puedan tener los jóvenes para formar una pareja actúan sobre la fecundidad a través de la tasa de formación de nuevos hogares.

Dejando a un lado, por ahora, los factores derivados del sistema de preferencias de los individuos —que también tiene que ver con razones económicas, por ejemplo a través de las pautas de consumoy ciñéndose a las cuestiones económicas más directas que afectan a la fecundidad, se pueden señalar dos de ellas, si bien íntimamente relacionadas: el mercado de trabajo y el acceso a la vivienda. Es ampliamente conocida la situación del mercado de trabajo en España en cuanto a las altas tasas de desempleo que ha venido padeciendo durante las dos últimas décadas de manera más acusada el grupo de población activa más joven. A esto hay que añadir que ese grupo, en su subdivisión femenina, ha sido especialmente afectado por el desempleo. También habría que señalar la precariedad de los empleos, tanto para hombres como para mujeres, aunque éstas lo sufren de manera más aguda. Ligado a esto, se encuentra la dificultad de obtener una vivienda, ya sea en propiedad o en alquiler. Estas dificultades, que afectan por igual ya se quiera formar un matrimonio o una pareja de hecho, son algunos de los obstáculos indirectos más importantes para la recuperación de la fecundidad, además de ser de los más difícilmente removibles. Son del tipo de los determinantes que, cuando menos, retrasan el calendario.

Hay otro tipo de factores económicos que inciden más directamente sobre el hecho de tener hijos o sobre el número que se tiene. Éstos se pueden agrupar en dos grandes apartados: en el de costes económicos directos que representa un hijo y en los costes de oportunidad, especialmente gravosos cuando trabajan ambos miembros de la pareja. El hecho de trabajar ambos miembros de la pareja influye de manera 
contradictoria, pues mientras resulta favorable para afrontar los costes directos (alimentación, educación, guarderías, cuidados en general), constituye un elemento que incrementa el coste de oportunidad para la pareja como unidad.

Las medidas tendentes a paliar los obstáculos a la fecundidad

El bajo nivel alcanzado por la tasa de fecundidad en España, ha logrado que los poderes públicos tomen conciencia de la situación y diseñen un conjunto de medidas que favorezcan la vida familiar principalmente en lo que hace a su conciliación con la vida laboraly animen o posibiliten el tener hijos a las parejas que lo deseen. Una gran mayoría de las medidas existían con anterioridad y lo que se ha hecho ahora es proceder a reformar la normativa y tratar de ajustarla a las nuevas condiciones.

No se va a hacer aquí un repaso de toda la legislación vigente en materia de familia, sino que la reflexión se circunscribirá al reciente Plan Integral de Apoyo a la Familia, aprobado en noviembre de 2001, principalmente en los aspectos más relacionados con la natalidad. Es un plan que, como se señala en su introducción, tiene como antecedentes más próximos la Ley de Conciliación de la Vida Familiar y Laboral de 1999, la reforma del IRPF de ese mismo año y algunas otras disposiciones posteriores respecto a la protección familiar por parte de la Seguridad Social. El Plan contempla cuatro grandes objetivos:

1. Incrementar la calidad de vida de las familias.

2. Fomentar la solidaridad intergeneracional.

3. Apoyar a la familia como garante de la cohesión social.

4. Prestar apoyo a las familias en situación de riesgo social y otras' situaciones especiales.

Estos objetivos se articulan en diez líneas estratégicas, cada una de ellas sustentada por medidas concretas:

1. Política fiscal y de rentas.

2. Mejora de las prestaciones de la Seguridad Social por hijo a cargo.

3. Conciliación de la vida familiar y laboral.

4. Política de vivienda.

5. Favorecer el acceso de las familias a las nuevas tecnologías. 
6. Revisión del Derecho de Familia.

7. Desarrollo de los servicios de orientación y/o mediación familiar.

8. Apoyo a las familias en situaciones especiales.

9. Fomento de la participación social y el acceso a la cultura de las familias.

10. Nueva Ley de protección a las familias numerosas.

De las diez líneas estratégicas, las más directamente relacionadas con la natalidad son las cuatro primeras y la última.

En la política fiscal y de rentas, a través de la segunda reforma del IRPF - cuyo Anteproyecto de Ley se presentó en el Consejo de Ministros de 19 de abril de 2002 - se incrementan las deducciones por hijo: 1.400 euros por el primero, 1.500 por el segundo, 2.200 por el tercero y 2.300 por el cuarto y superiores. (Antes eran 1.200 euros por el primero y el segundo y 1.800 por los siguientes). Se introducen dos nueva deducciones: una de 1.200 euros por el cuidado de menores de tres años (sin necesidad de justificación), así como otra de igual cuantía para las madres trabajadoras en los tres años siguientes al nacimiento o la adopción del hijo. También se implementa una ayuda especial a las familias numerosas.

Respecto a las medidas encuadradas en las prestaciones de la Seguridad Social, se incrementan las asignaciones por hijos a cargo que tengan más de 18 años y padezcan alguna discapacidad y se amplía el límite para tener derecho a las prestaciones por hijos menores de 18 años. Se prevé aumentar la cuantía del pago único por nacimiento de un hijo y extender determinados beneficios a los casos de adopción $\mathrm{y}$ acogimiento.

En cuanto a la conciliación de la vida familiar y laboral, se desarrolla el Reglamento de la Ley de Conciliación de la Vida Familiar y Laboral de las personas trabajadoras. En este sentido, se ha publicado un Real Decreto en desarrollo de dicha ley, que favorece la posibilidad de disfrutar el permiso de maternidad y trabajar a tiempo parcial simultáneamente. Se pretende favorecer la contratación posterior a la maternidad, establecer una bonificación empresarial respecto al pago a la Seguridad Social para el caso de contratación de madres y padres solteros, así como para contingencias comunes durante la suspensión del contrato de trabajo por maternidad. También se pretende elaborar un «Código de buenas prácticas» (en colaboración con las empresas y agentes sociales), mejorar la normativa sobre educación infantil e incrementar estos servicios hasta cubrir con plazas públicas la demanda existente para niños entre cero y tres años. 
La política de vivienda contempla como una de sus medidas dirigir la oferta de viviendas de protección oficial a las familias con menores niveles de ingresos e incluir el número de hijos como criterio preferencial al optar a una de estas viviendas. Esta idea se desarrolla en el Real Decreto que regula el nuevo Plan de Vivienda 2002-2005, aprobado en Consejo de Ministros. Se apoya el acceso a las viviendas protegidas por parte de las familias con ingresos menores a 5,5 veces el salario mínimo interprofesional, que, en el caso de tratarse de familias numerosas, se incrementa en un 20\%. Se implementan sistemas de ayuda para financiar la entrada en la compra de la vivienda para familias en función de sus ingresos, pero que no rebasen 3,5 veces el salario mínimo interprofesional. Entre las ayudas directas de carácter especial a la compra de una vivienda, los jóvenes menores de 35 años recibirán una ayuda de 35.000 euros para la entrada, que será acumulable a otras ayudas a las que pudieran tener derecho.

Por lo que hace a la Nueva Ley de Protección de las Familias Numerosas, existe en el Senado una ponencia en el marco de la Comisión de Trabajo y Asuntos Sociales, que está analizando la situación de este tipo de familias, recabando la opinión de expertos, representantes políticos y asociaciones familiares en orden a realizar propuestas para elaborar un nuevo texto que sustituya al actualmente vigente.

\section{Conclusiones}

Este breve y no exhaustivo repaso a las medidas que se han implementado recientemente o que se tiene intención de implementar, se ha ceñido a las que más afectan a la natalidad, ya sea directa o indirectamente. Pero, con independencia de su oportunidad y eficacia, hay que empezar por hacer una observación de carácter general, que no por insistentemente reiterada debe pasarse por alto, y es la dificultad de llevar a cabo un plan como es el de apoyo a la familia, cuando dicho plan no tiene dotación económica. Se trata más bien de un plan de acciones de gobierno o de una declaración de intenciones, pues un verdadero conjunto de medidas que, de llevarse a cabo, tendrían un coste económico, debiera llevar tras de sí disposiciones concretas, avaladas por su correspondiente dotación presupuestaria. Y esto sólo se encuentra en un reducido número de aspectos, principalmente aquellos que tienen que ver con la política fiscal y de vivienda.

Otro importante aspecto a destacar es que al enumerar las distintas medidas se pone el acento en las familias ya constituídas y mucho 
menos en apoyar la formación de las nuevas. Ambas cosas son necesarias $y$, por tanto, no excluyentes, pero si se tienen en cuenta los indicadores demográficos actuales - con la nupcialidad como uno de los factores que más ha contribuído en los noventa a deprimir la tasa de fecundidad-, el favorecer la formación de nuevos hogares sería una medida de la que cabría esperar un repunte de la fecundidad a medio plazo. Esto pasa, indudablemente, por una decidida política de creación de empleo, por supuesto no precario, y una política de vivienda, que esa sí parece que se ha tenido en cuenta, si bien orientada preferentemente hacia las familias con hijos.

Las subvenciones por el nacimiento de un hijo, las deducciones en el impuesto sobre la renta por hijos a cargo y las medidas similares tienen como finalidad ayudar económicamente a las personas que se deciden a tener hijos, pero sus cuantías no son determinantes a la hora de tomar la decisión. Siendo muy optimistas se puede pensar que ayudan en esa dirección y la facilitan algo, pero en términos de estricto cálculo de costes no llegan a sufragar una mínima parte de los gastos originados. Por tanto, la decisión se toma en función de otros parámetros, muchos de ellos de corte social o psicológico. En esa línea, parece preferible hablar de incentivos sociales a la natalidad, que englobarían también los económicos.

Pero ¿cuáles habrían de ser esos incentivos sociales a la natalidad?. La respuesta no es fácil ni unívoca, en la medida en que los determinantes sociales de la fecundidad están estrechamente vinculados al sistema de valores (Van de Kàa, 1998) y de ahí, que sean cambiantes en el tiempo y entre sociedades. No obstante, se hace necesario un cambio en la valoración social del cuidado de los hijos. Y eso exige cambios en la dimensión pública y privada. Por cambios en la esfera privada se entiende una profundización en ciertas pautas -aún sólo emergentes- que tienen que ver con el reparto equitativo de las responsabilidades en el seno de la familia respecto a las tareas referidas a la crianza de los niños, su cuidado y la asunción del peso de las responsabilidades que conlleva un hogar.

Los cambios en el ámbito público han de ir acompañados de la concienciación por parte de los poderes públicos y los agentes sociales - empleadores en primer término- de la maternidad/paternidad como algo cuyos costes deben afrontarse colectivamente y no como un coste particular de las familias, en cuyo caso es muy difícil que pueda repuntar la fecundidad. Dicho coste, tal vez pueda percibirse gravoso a muy corto plazo, pero con una perspectiva más amplia constituye un asunto socialmente ineludible. Esta percepción ha de ir calando en la sociedad 
y, por tanto, han de arbitrarse los mecanismos para resolver la disyuntiva que hoy se plantea a las parejas que quieren tener un hijo. Para ello deben mejorarse las condiciones para el trabajo a tiempo parcial, los permisos laborales por el nacimiento de un hijo - tanto para la madre como para el padre-, la red de guarderías, tratar de compaginar mejor los horarios laborales y las actividades escolares, y un largo etcétera de medidas que faciliten el ser padres sin que se resienta la vida profesional de alguno de los miembros de la pareja o que, incluso, haya que renunciar a ella. Tales medidas tienen costes económicos y pueden entrar en conflicto con intereses empresariales, amén de otros conflictos en el ámbito público entre lo que, sin duda, se considera deseable y lo que son las posibilidades reales en términos presupuestarios. Pero es un esfuerzo que hay que afrontar e ir poniendo en marcha paulatinamente, a efectos de acercarnos a la media de la Unión Europea en lo que a gasto social se refiere, y, más concretamente, en cuanto a gasto en protección a la familia. En ese sentido, las voces de alarma demográfica que han empezado a sonar pueden servir de acicate para ese cambio social, consistente en una modificación de conductas, pero ésta sólo se producirá si se modifica la valoración social de la reproducción.

\section{Referencias bibliográficas}

Allan, G. (2001) "Family Diversity and Change in Britain and Western Europe", Journal of Family Issues, pp. 819-837.

ARIES, PH., (1981) "Two successive motivations for the declining birth rate in the West» en Höhn, C., Mackensen, R., (eds.) Determinants of Fertility Trends: Theories Reexamined, Liège, pp. 123-130.

BECKER, G., (1980) «El enfoque económico del comportamiento humano», Información Comercial Española, enero, pp. 11-18.

Bosveld, W. (1996) The Ageing of Fertility in Europe, Amsterdam, Thesis Publishers.

CALDwell, J. (1981) “The Wealth Flows Theory of Fertility Decline» en Höhn, C., Mackensen, R. (eds.), Determinants of Fertility Trends; Theories Re-examined, Liège, pp. 169-188.

DELGADO, M. (2000) “Los componentes de la fecundidad: su impacto en la reducción del promedio de hijos por mujer en España", Economistas, 86, pp. 23-34.

GustafsSON, S. (2001) “Optimal age at motherhood. Teoretical and empirical considerations on postponement of maternity in Europe", Journal of Population Economics, 14, pp. 225-247.

Leibenstein, H., (1981) "Economic Decision Theory and Human Fertility Behavior: A Speculative Essay", Population and Development Review, 7,3, pp. 381-400. 


\section{Margarita Delgado}

282

VAN DE KÀA, J.D. (1998) «Postmodern Fertility Preferences: from Changing Value Orientation to New Behaviour", Working Papers in Demography, 74, The Australian National University.

\section{Notas}

1 La natalidad pone en relación el volumen de nacimientos en una población con la cifra total de la misma, mientras que la fecundidad pone en relación los nacimientos con el efectivo de mujeres en edad de procrear. 